\title{
A fisioterapia nas unidades de terapia intensiva neonatal
} The physiotherapy in the neonatal intensive care units

Francisco Eudison da Silva Maia ${ }^{1}$

\begin{abstract}
RESUMO
A intervenção do profissional fisioterapeuta nas Unidades de Terapia Intensiva Neonatal (UTIN) é relativamente recente e tem se apresentado como uma conduta de singular importância. Conforme a portaria 3.432 do Ministério da Saúde, as Unidades de Terapia Intensiva, se estendendo às voltadas aos cuidados em neonatos, devem contar com assistência fisioterapêutica durante um mínimo de 12 horas por dia, por considerar que a atuação deste profissional pode diminuir as complicações, o período de internação e, consequentemente, os custos hospitalares. A principal função da assistência do profissional em questão é promover o desenvolvimento neuropsicomotor favorável aos prematuros, prevenir e reduzir possíveis complicações respiratórias, melhorar a função pulmonar, sempre buscando uma evolução constante do paciente. Palavras-chave: fisioterapia; unidades de terapia intensiva neonatal; recém-nascido.

ABSTRACT

The professional physiotherapist's intervention in the Neonatal Intensive Care Unit (NICU) it is relatively recent and has been proving to be of singular importance. According to ordinance 3.432 of the Brazilian Ministry of Health, the Intensive Care Unit, including those dedicated to the care of newborns, should provide physical therapy assistance during at least 12 hours a day, as this professional's performance is considered to reduce complications and the hospitalization period and, consequently, the hospital costs. The physical therapy professional's main role is to promote a favorable neuromotor development to premature neonates, to prevent and to reduce possible breathing complications, to improve the lung function, always aiming for the patient's constant evolution.
\end{abstract}

Keywords: physiotherapy; neonatal care intensive units; infant newborn.

\section{INTRODUÇÃO}

Os recém-nascidos $(\mathrm{RN})$ em condição de prematuridade apresentam diversas situações de risco por possuírem instabilidade fisiológica e/ou hemodinâmica. ${ }^{1-3}$

Após o nascimento, o ambiente extrauterino condiciona o neonato a uma série de adaptações à vida, que incluem a maturação morfofisiológica e bioquímica do parênquima pulmonar e suas estruturas correlacionadas. Neste contexto, os prematuros apresentam uma maior vulnerabilidade por não possuírem uma função pulmonar adequada, necessitando frequentemente do uso da oxigenoterapia, visando assegurar as trocas gasosas necessárias à vida. ${ }^{4}$

Quanto mais baixos o peso e a idade gestacional, maior a probabilidade de riscos biológicos para os RNs, podendo desenvolver problemas com sequelas de difícil reversão, como os relacionados ao desenvolvimento físico, cognitivo, de aprendizagem e comportamental. ${ }^{5}$

\section{DISCUSSÃO}

Ao analisarmos as internações nas Unidades de Terapia Intensiva Neonatal (UTIN), podemos observar que os números decorrentes dos diagnósticos de prematuridade, peso muito baixo ao nascer, anoxia perinatal, má formação, entre outros, vêm crescendo cotidianamente. Frente a esta realidade, a implantação desse tipo de ambiente e os avanços tecnológicos têm acarretado uma potencialização nos diagnósticos precoces e uma maior chance de sobrevivência aos recémnascidos pré-termo com problemas de saúde. ${ }^{2}$

Mesmo frente às constantes evoluções nos ambientes das UTINs, a prematuridade provoca várias intercorrências no processo de crescimento e desenvolvimento dos recém-nascidos pré-termo (RNPT). Embora apresente uma maior sobrevida, uma intervenção prolongada nas unidades de terapia intensiva pode promover ou potencializar uma serie de agravos ao desenvolvimento neuro-sensório-motor.

${ }^{1}$ Universidade Potiguar (UnP) - Mossoró (RN), Brasil.

Contato: eudisonmaia@yahoo.com.br

Recebido em 04/03/2015. Aceito para publicação em 23/06/2015. 
Sob esta ótica, a presença e atenção à saúde de uma equipe multiprofissional se tornam indispensáveis. ${ }^{6}$

Somada à equipe multiprofissional, a fisioterapia nas UTINs é uma modalidade de terapia relativamente nova. Contudo, os avanços da assistência fisioterapêutica ao recém-nascido, em especial aos que necessitam de cuidados intensivos, são capazes de proporcionar um aumento na sobrevida e reduzir sequelas sistêmicas. ${ }^{2}$

A intervenção clínica deste profissional visa evitar agravamento de síndromes aspirativas, na síndrome do desconforto respiratório, pneumonias, atelectasias, na prevenção de complicações provenientes da ventilação mecânica, secreções nas vias aéreas, nos casos com evoluções desfavoráveis à gasometria e/ou ao exame radiológico, sinais indicativos de possíveis problemas com a depuração ciliar, reduzir as incidências de complicações pulmonares, atelectasias pós-extubação e impactar positivamente o prognóstico, entre outros. ${ }^{7}$

Em algumas situações, a fisioterapia tem se mostrado uma intervenção prioritária, por promover a otimização da função respiratória, a facilitação correlacionada às trocas gasosas, a otimização da relação ventilação-perfusão, a manutenção da permeabilidade das vias aéreas e o desmame da ventilação mecânica e da oxigenoterapia. ${ }^{8}$ Nesse ínterim, se houver uma assistência adequada de um fisioterapeuta, os agravantes para os neonatos graves podem ser minimizados, com evoluções promissoras. ${ }^{9}$

É importante monitorar o desenvolvimento dos RN sobre os aspectos do profissional em apreço nos ambientes das UTINs, de forma a detectar precocemente possíveis desvios patogênicos, visando prevenir, intervir ou minimizar sequelas, pois quanto mais precoce for o diagnóstico de comprometimentos e a intervenção, menor será o impacto na vida futura da criança. ${ }^{10,11}$

Mesmo frente aos dados, Silva et al. ${ }^{12}$, em seu estudo, coloca que o tema em questão está em ascensão e que há uma evidente tendência ao crescimento da submissão de projetos de pesquisa na área de Fisioterapia Neonatal e Pediátrica. Mas, mesmo dentro dessa tendência, tais submissões ainda representam muito pouco. Para o autor em questão, as pesquisas voltadas para a área em apreço ainda têm um longo caminho em termos de pesquisa científica.

\section{CONSIDERAÇÕES FINAIS}

A cobertura fisioterapêutica nas UTINs se faz necessário em virtude da população de RNs em risco, que necessitam de uma assistência que possa prevenir os transtornos em seu desenvolvimento neuropsicomotor.

Aparte desse manuscrito, foi possível identificar que a abordagem fisioterapêutica, embora aceita e tratada como essencial no atendimento da UTINs, ainda carece de pesquisas que comprovem sua eficácia, com resultados mais conclusivos.

Porém, mesmo com a ressalva, foi possível obter um panorama da importância da intervenção da fisioterapia nas Unidades de Terapia Intensiva Neonatal. A literatura é categórica ao destacar a importância desta área para redução ou prevenção dos comprometimentos dos neonatos nas UTINs. Ressalta, ainda, que deve ser iniciada preco- cemente, para reduzir o tempo de permanência hospitalar, com consequente redução de gastos.

\section{REFERÊNCIAS}

1. Selestrin CC, Oliveira AG, Ferreira C, Siqueira AAF, Abreu LC, Murad N. Avaliação dos parâmetros fisiológicos em recém-nascidos pré-termo em ventilação mecânica após procedimentos de fisioterapia neonatal. Rev Bras Crescimento Desenvolv Hum. 2007;17(1):146-55.

2. Vasconcelos GAR, Almeida RCA, Bezerra AL. Repercussões da fisioterapia na unidade de terapia intensiva neonatal. Fisioter Mov. 2011;24(1):65-73.

3. Zani AV, Tonete VLP, Parada CGL. Cuidados a recémnascidos de baixo peso por equipes de saúde da família: revisão integrativa. Rev Enferm UFPE on line. 2014;8(5):1347-56.

4. Siqueira VSA, Alves VH, Barbosa MTSR, Rodrigues DP, Vieira BDG, Silva LA. Indicadores de qualidade na assistência ventilatória em um hospital universitário: saber fazer na enfermagem. Rev Enferm UFPE on line. 2014;8(4):797-807.

5. Vieira MEB, Linhares MBM. Desenvolvimento e qualidade de vida em crianças nascidas prétermo em idades pré-escolar e escolar. J Pediatr. 2011;87(4):281-91.

6. Sassá AH, Schmidt KT, Teston EF, Marcon SS. A prática alimentar e o crescimento do bebê nascido com muito baixo peso ao longo do primeiro ano de vida estudo descritivo. Online Braz J Nurs [Internet]. 2010 [acesso em 01 fev. 2015];9(2). Disponível em: http:// www.objnursing.uff.br/index.php/nursing/article/ view/j.1676-4285.2010.3074/691

7. Johnston C, Zanetti NM, Comaru T, Ribeiro SNS, Andrade LB, Santos SLL. I Recomendação Brasileira de fisioterapia respiratória em unidade de terapia intensiva pediátrica e neonatal. Rev Bras Ter Intensiva. 2012;24(2):119-29.

8. Nicolau CM, Lahóz AL. Fisioterapia respiratória em terapia intensiva pediátrica e neonatal: uma revisão baseada em evidências. Pediatria. 2007;29(3):216-21.

9. Nicolau CM, Costa APBM, Hazime HO, Krebs VLJ. Desempenho motor em recém-nascidos pré-termo de alto risco. Rev Bras Crescimento Desenvolv Hum. 2011;21(2):327-34.

10. Moreira EG, Grave MTQ. Avaliação do desenvolvimento motor de crianças prematuras nascidas em uma pequena cidade do vale do rio dos sinos. Rev Destaques Acad. 2014;6(3):64-71.

11. Brasil. Ministério da Saúde. Atenção à saúde da criança de 0 a 12 anos. Porto Alegre: Hospital Nossa Senhora da Conceição; 2009. p. 200.

12. Silva COS, Gardenghi G. Análise quantitativa da submissão de projetos de pesquisa científica realizados em Fisioterapia Pediátrica e Neonatal cadastrados no sistema CEP/CONEP de 2002 a 2010. ASSOBRAFIR Ciência. 2012;3(1):33-43. 